\title{
1 Selective attention and decision-making have separable neural bases in
}

\section{2 space and time}

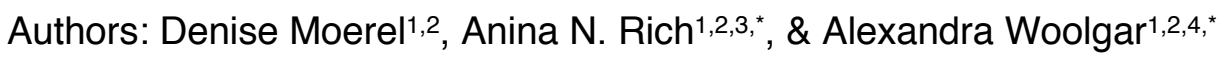

* Equal contribution

1. Department of Cognitive Science, Macquarie University, Sydney, Australia

2. Perception in Action Research Centre, Macquarie University, Sydney, Australia

3. Centre for Elite Performance, Expertise and Training, Macquarie University, Sydney, Australia

4. MRC Cognition and Brain Sciences Unit, University of Cambridge, Cambridge, United Kingdom

\section{ABSTRACT}

Attention and decision-making processes are fundamental to cognition. However, they are usually experimentally confounded, making it impossible to link neural observations to specific processes. Here we separated the effects of selective attention from the effects of decisionmaking in human observers using a two-stage task where the attended stimulus and decision were orthogonal and separated in time. Multivariate pattern analyses of multimodal neuroimaging data revealed the dynamics of perceptual and decision-related information coding through time (magnetoencephalography (MEG)), space (functional Magnetic Resonance Imaging (fMRI)), and their combination (MEG-fMRI fusion). Our MEG results showed an effect of attention before decision-making could begin, and fMRI results showed an attention effect in early visual and frontoparietal regions. Model-based MEG-fMRI fusion suggested that attention boosted stimulus information in frontoparietal and early visual regions before decision-making was possible. Together, our results suggest that attention affects neural stimulus representations in frontoparietal regions independent of decision-making. 


\section{INTRODUCTION}

Selective attention is a mechanism that prioritises relevant information from amongst competing sensory input. With clear behavioural benefits to attending to particular locations in space (Pestilli \& Carrasco, 2005; Posner, 1980) or to particular visual features (Sàenz et al., 2003; White \& Carrasco, 2011), there is strong evidence that attention affects perception (Carrasco, 2011). However, in cognitive neuroscience experiments, the selection and maintenance of relevant stimulus information is commonly confounded with decision-making processes that pertain to the selected input. We address this ambiguity by separating the effects of attentional selection and maintenance on the neural processing of information from the effects of decision-making to study of the spatiotemporal correlates of each process separately.

The adaptive coding hypothesis (Duncan, 2001) proposes a framework for how selective attention could affect stimulus processing. According to this hypothesis, single cells in frontoparietal cortex can flexibly adapt their response to code different information as needed for the task, which provides a potential source of bias for more selective brain regions (Desimone \& Duncan, 1995). Accordingly, single cell recordings in non-human primates show task-dependent neural tuning profiles in prefrontal cortex (Rao et al., 1997; Sigala et al., 2008; Stokes et al., 2013), with an emphasis on coding information that is currently relevant for the task (Kadohisa et al., 2013). In humans, a network of frontoparietal regions also responds with a profile consistent with adaptive coding. We will use the term 'Multiple Demand' (MD) regions (Duncan, 2010; Duncan \& Owen, 2000) to refer to this frontoparietal network, but similar networks have been referred to as the 'frontoparietal control system' (Vincent et al., 2008), 'task positive network' (Fox et al., 2005), and 'task activation ensemble' (Seeley et al., 2007). Functional Magnetic Resonance Imaging (fMRI) studies in human participants show that the MD regions respond to a wide range of 
cognitively demanding tasks (Assem et al., 2020; Dosenbach et al., 2006; Duncan \& Owen, 2000; Fedorenko et al., 2013).

More specific evidence for an adaptive response in the MD regions comes from studies that use multivariate pattern analyses (MVPA) to test for the contents of the neural representations, using the patterns of response across voxels (fMRI) or sensors (MEG) (see Grootswagers et al., 2016; Haynes, 2015; Hebart \& Baker, 2017). Studies using this technique on $\mathrm{fMRI}$ data show that multivariate patterns of activity code for a range of taskrelevant information in different contexts (Woolgar et al., 2016), with single MD voxels reused to code multiple task features between tasks (Jackson \& Woolgar, 2018). In addition, Woolgar and colleagues (2015) found stronger visual object coding in the MD cortex for cued (i.e., attended, task-relevant) objects relative to equivalent distractor objects, and Jackson and colleagues (2016) reported stronger MD coding of cued compared to equivalent distractor features of the same object. Moreover, MD coding of cued information can be selectively impaired by non-invasive stimulation the right dorsolateral prefrontal cortex, one of the nodes in the MD network (Jackson et al., 2020). In parallel, recent magnetoencephalography (MEG) studies have shown the coding of a cued feature at a cued location is sustained over time, while information coding for distractor information is not sustained (Battistoni et al., 2018; Goddard et al., 2019). What we do not know, however, is whether this difference in information coding between cued and distractor stimuli is driven by the attentional selection and maintenance of relevant information or, at least partially, by decision-making processes pertaining to that information. In most paradigms, including those used in the studies outlined above, attention is manipulated by asking participants to respond to the cued (but not the distractor) stimulus. As decisions pertain to the cued information only, attending to a stimulus and making a decision about the stimulus could both contribute to previous findings. 
Underscoring the importance of this confound, there is clear evidence that decisions can drive decoding, although these studies do not explicitly investigate the role of attention. For instance, decisions can be decoded from early visual areas (Löffler et al., 2019; Rens et al., 2017), as well as from frontoparietal cortex (Löffler et al., 2019). In these paradigms, the decision was not driven by visual information, as participants had to make a choice between multiple options. Bode and colleagues (2012) found perceptual decisions could be decoded from 140-180ms post-stimulus onwards from pure noise images. This is a similar timecourse as the effect of attention on stimulus coding (Goddard et al., 2019). Thus, decisions can be decoded in similar areas of the brain, and with a similar time-course as effects of attention, further complicating interpretation of the extant literature.

There is, however, some evidence suggesting that effects of attention can occur in the absence of decision-making processes. For example, in a univariate fMRI study, Hon and colleagues (2006) showed activation in the MD regions in response to changes in cued streams, but not in distractor streams of stimuli. This occurred even when the participant did not have to respond or did an orthogonal task. This study links attention to overall changes in blood flow to MD regions but did not investigate what information was coded about the attended and unattended stimuli.

Here, we used MVPA of MEG and fMRI data to address 3 research questions. First, we asked whether attention affects the coding of stimulus information when it is separated from decision-making processes in time. We used the high temporal resolution of MEG in combination with a 2-stage task to dissociate the coding of attended and unattended visual information from coding of decision-related information. Our data showed that relevant information was prioritised during the first stage, showing a clear effect of selecting and maintaining specific features before participants could make a decision. Second, we used fMRI to ask what type of information the MD regions hold. The results showed that the MD regions preferentially code visual information when it is cued as relevant, compared to when 
103

104

105

106

107

108

109

110

111

112

113

114

115

116

117

118

119

120

121

122

123

124

125

126

the same visual information is a distractor, as well as the orthogonal decisions about the stimuli needed to generate a response. This suggests the MD regions are involved both in supporting selective attention and in decision-making. Finally, we used model-based MEGfMRI to formally combine the data from the two imaging modalities and examine the timecourse with which the MD regions preferentially coded for cued compared to distractor information during the first phase of the trial. The results suggest that the MD regions selectively code for cued visual information before decision-making begins in this task. These data show that while similar brain regions are involved in both attention and decisionmaking, the two processes are separable with distinct spatiotemporal neural correlates.

\section{MATERIALS AND METHODS}

\subsection{MEG acquisition and analysis}

\subsubsection{Participants}

The first part of this study consisted of a behavioural training session and a MEG session carried out at Macquarie University (Sydney, Australia). We tested 31 healthy volunteers in the behavioural training session. Of these, 21 performed well enough to participate in the MEG session (at least $90 \%$ accuracy in the final run of the training session). The MEG data from 1 participant were excluded due to a failure to complete the experiment, resulting in a final MEG sample of 20 participants (14 female/6 male, 18 righthanded/2 lefthanded, mean age $=25.8$ years, $S D=5.1$ ). Participants received $\$ 15$ AUD for participation in the training session and $\$ 30$ AUD for participation in the MEG session. The study was approved by the Macquarie University Human Research Ethics Committee, and all participants provided informed consent. 


\subsubsection{Stimuli and experimental procedure}

MEG session

129 Participants were instructed keep fixation at a central bullseye throughout the experiment and were cued to attend to either blue or orange at the start of each block (32 trials). Figure 1 shows the stimuli and trial procedure. First, approximately equiluminant blue and orange oriented lines within a circular window were overlaid at fixation for $150 \mathrm{~ms}$ on a mid-grey background (Figure 1A). Participants attended to the lines of the cued colour, while ignoring the lines of the other colour. After a 500ms blank screen, a black comparison line was presented for $200 \mathrm{~ms}$. The task was to determine which way the cued lines had to be rotated to match the orientation of the comparison line. After the comparison line was presented, there was a 500ms blank screen, and then the response screen, consisting of arrows showing either a clockwise or an anti-clockwise rotation, was shown until a response was given. If participants did not respond within 3 seconds, the next trial started. Participants pressed one of two buttons to indicate whether the rotation shown on the response screen was correct or incorrect. We used this response screen to separate the correct response button from the rotation direction decision, ensuring that rotation direction decoding could not be driven by motor preparation. The mapping of the buttons to indicate 'correct' or 'incorrect' was counterbalanced across participants. Participants received feedback on every trial; at the end of each trial, the white part of the fixation bullseye turned green (when correct) or red (when incorrect) for $250 \mathrm{~ms}$ to give feedback on accuracy.

148 (DVA), a spatial frequency of 2 cycles/degree, were 2 possible colours (blue; RGB = 239, $159,115$ and orange; $R G B=72,179,217)$, and were phase randomised (Figure $1 \mathrm{~A})$. The comparison line had a length of 0.8 DVA and a width of 0.1 DVA. The response mapping arrows were 1.5 DVA, and the central fixation bullseye had a diameter of 0.4 DVA. There 
into 2 pairs: $22.5^{\circ}$ was paired with $112.5^{\circ}$, and $67.5^{\circ}$ was paired with $157.5^{\circ}$ (Figure $1 \mathrm{~B}$ ).

When the cued orientation was from one pair, the distractor and comparison orientation

A

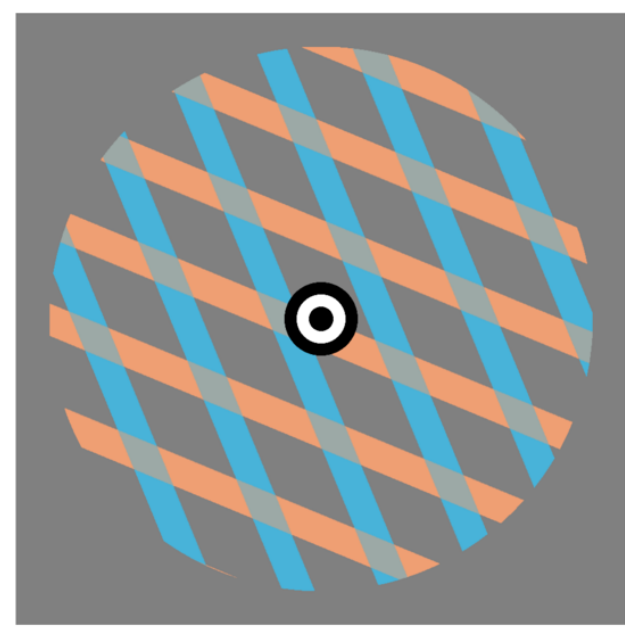

C

Cued \&

distractor orientation

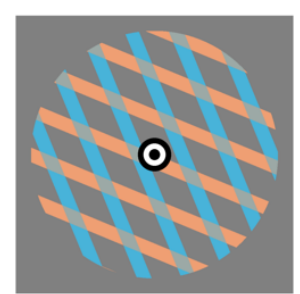

$150 \mathrm{~ms}$

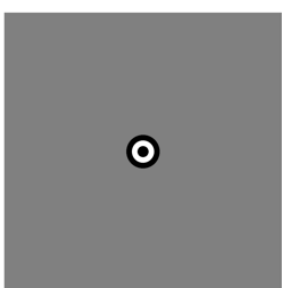

$500 \mathrm{~ms}$
B

\begin{tabular}{|l|l|l|l|l|}
\hline & $\begin{array}{l}\text { Cued } \\
\text { orientation }\end{array}$ & $\begin{array}{l}\text { Distractor } \\
\text { orientation }\end{array}$ & $\begin{array}{l}\text { Rotation } \\
\text { direction }\end{array}$ & $\begin{array}{l}\text { Comparison } \\
\text { orientation }\end{array}$ \\
\hline 1 & $22.5^{\circ}$ & $67.5^{\circ}$ & Clockwise & $67.5^{\circ}$ \\
\hline 2 & $22.5^{\circ}$ & $67.5^{\circ}$ & Anti-clockwise & $157.5^{\circ}$ \\
\hline 3 & $22.5^{\circ}$ & $157.5^{\circ}$ & Clockwise & $67.5^{\circ}$ \\
\hline 4 & $22.5^{\circ}$ & $157.5^{\circ}$ & Anti-clockwise & $157.5^{\circ}$ \\
\hline 5 & $112.5^{\circ}$ & $67.5^{\circ}$ & Anti-clockwise & $67.5^{\circ}$ \\
\hline 6 & $112.5^{\circ}$ & $67.5^{\circ}$ & Clockwise & $157.5^{\circ}$ \\
\hline 7 & $112.5^{\circ}$ & $157.5^{\circ}$ & Anti-clockwise & $67.5^{\circ}$ \\
\hline 8 & $112.5^{\circ}$ & $157.5^{\circ}$ & Clockwise & $157.5^{\circ}$ \\
\hline 9 & $67.5^{\circ}$ & $22.5^{\circ}$ & Anti-clockwise & $22.5^{\circ}$ \\
\hline 10 & $67.5^{\circ}$ & $22.5^{\circ}$ & Clockwise & $112.5^{\circ}$ \\
\hline 11 & $67.5^{\circ}$ & $112.5^{\circ}$ & Anti-clockwise & $22.5^{\circ}$ \\
\hline 12 & $67.5^{\circ}$ & $112.5^{\circ}$ & Clockwise & $112.5^{\circ}$ \\
\hline 13 & $157.5^{\circ}$ & $22.5^{\circ}$ & Clockwise & $22.5^{\circ}$ \\
\hline 14 & $157.5^{\circ}$ & $22.5^{\circ}$ & Anti-clockwise & $112.5^{\circ}$ \\
\hline 15 & $157.5^{\circ}$ & $112.5^{\circ}$ & Clockwise & $22.5^{\circ}$ \\
\hline 16 & $157.5^{\circ}$ & $112.5^{\circ}$ & Anti-clockwise & $112.5^{\circ}$ \\
\hline
\end{tabular}

Comparison

Response

orientation

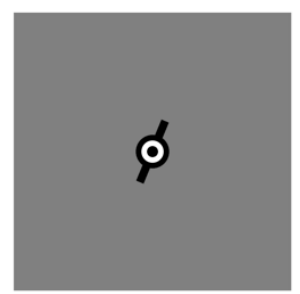

$200 \mathrm{~ms}$

screen

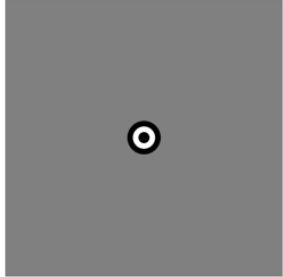

$500 \mathrm{~ms}$

Figure 1. Stimuli and design. The stimulus, shown in A, consisted of oriented blue and orange lines within a circular window. Participants attended to the lines of one colour, while ignoring the lines of the other colour. B shows the 16 possible trial types, determined by a combination of cued orientation, distractor orientation, and rotation direction. The comparison orientation was not used in the analysis but is shown for completeness. The cued orientation, distractor orientation, and rotation direction are orthogonal dimensions in this design, only within the 2 pairs separately (top half and bottom half of the table). 
example of a trial sequence. Participants were cued to a particular colour for a block of 32 trials. On each trial, the cued and distractor oriented lines were shown for $150 \mathrm{~ms}$ followed by $500 \mathrm{~ms}$ delay, and then the comparison orientation was shown for $200 \mathrm{~ms}$. After another $500 \mathrm{~ms}$ delay, the response screen appeared until a response ('correct' vs 'incorrect') was given, or until the $3 s$ timeout. In this trial, for example, if the cued colour was blue, the participant would have to decide whether the blue lines need to rotate clockwise or anticlockwise to match the comparison orientation. At the response screen, which in this trial shows clockwise, the correct response would be the button indicating 'correct'.

Cued orientation (4) $\mathrm{x}$ distractor orientation (2) $\mathrm{x}$ rotation direction (2) $\mathrm{x}$ response screen (2) were counterbalanced within each block, and the cue colour was counterbalanced over blocks. Participants were instructed which colour to attend at the start of each block of 32 trials, and the cue colour switched between blocks. Participants completed 16 experimental runs, with 2 blocks per run (one block of 32 trials per cue colour). One participant completed 14 instead of 16 experimental runs. In addition to the feedback on every trial, participants received feedback on their accuracy at the end of each block. The order of the trial types was randomised within each block. To increase the number of correct trials, any trial on which the participant made an error was presented again later in the block. This was done a maximum of two times per block, and only if it was not the last trial in the block. We replaced error trials with successful retakes in the analysis, thus increasing the number of correct trials available for the decoding analysis. We did not record eye movements, but to influence decoding accuracy, eye-movements during the trials would have to systematically vary between the different conditions within each pair (see Figure 1B). This seems unlikely as all cued and distractor orientations were presented at fixation with no variance between conditions.

\section{Training session}

Due to the challenging task, participants completed a separate behavioural training session before doing the task in the MEG. The training session consisted of 3 parts. First, participants practiced the rotation task without selection. Only lines of the cued colour were 
shown, and the task was slower: the cued stimulus and comparison line were both presented for $500 \mathrm{~ms}$ instead of $150 \mathrm{~ms}$ and $200 \mathrm{~ms}$ respectively. Next, the selection element was introduced. Both the cued and the distractor colour lines were shown, with the timing of the task again slowed as in part 1 of the training. Finally, the task was presented at the same speed as used in the MEG session: the cued stimulus was shown for $150 \mathrm{~ms}$ and the comparison line was presented for $200 \mathrm{~ms}$. Feedback was given at the end of each trial by the white part of the fixation bullseye turning green (correct) or red (incorrect) for $250 \mathrm{~ms}$. At the end of each block, participants also received feedback about their overall accuracy for that block. cued colour. The number of runs per training part was adjusted flexibly depending on task performance. When participants had finished at least 2 runs and achieved an average accuracy of at least $90 \%$ for the previous run, they moved onto the next part of training. invited to participate in the MEG session.

\subsubsection{MEG acquisition}

215 Participants performed the task in a supine position. The MEG signal was continuously sampled at $1000 \mathrm{~Hz}$ on a whole-head MEG system with 160 axial gradiometers inside a

217 magnetically shielded room (Model PQ1160R-N2, KIT, Kanazawa, Japan). All MEG recordings were obtained at the KIT-Macquarie Brain Research Laboratory, Sydney, Australia. Recordings were filtered online between $0.03 \mathrm{~Hz}$ and $200 \mathrm{~Hz}$. To track head movements during the experiment, participants were fitted with a cap with 5 marker coils. 
224

225

226

227

228

229

230

231

232

233

234

235

236

237

238

239

240

241

242

243

244

245

246

247

248

249

photodiode responses were used to determine the onset of the trial. We adjusted the stimuli to correct for distortions introduced by the projector. The stimuli were presented using the Psychtoolbox extension in MATLAB (Brainard, 1997; Kleiner et al., 2007; Pelli, 1997).

Participants used a 2-button response pad to respond on each trial.

\subsubsection{Pre-processing}

The data were pre-processed using the FieldTrip extension in MATLAB (Oostenveld et al., 2011). The recordings were sliced into $3100 \mathrm{~ms}$ epochs; from $100 \mathrm{~ms}$ before the onset of the first stimulus until $3000 \mathrm{~ms}$ after stimulus onset. The signal was then down sampled to $200 \mathrm{~Hz}$. Error trials that had a successful retake were exchanged for the correct retake. To keep the counterbalancing of all conditions intact, we included the few error trials that did not have a successful retake in the analysis $(0.33 \%$ of trials on average).

\subsubsection{Decoding analysis}

We used MVPA to determine whether there was information present in the pattern of activation for each timepoint about: 1) the cued orientation; 2) the distractor orientation; and 3) the rotation direction. Comparing the coding of the cued and distractor orientations allowed us to determine whether attended visual information was preferentially coded, while coding of the rotation direction reflected the decision participants had to make about the stimulus. Critically, the cued orientation, distractor orientation, and rotation direction were all orthogonal dimensions, allowing us to investigate the coding of each of these separately. For each timepoint, we trained a Support Vector Machine (SVM) classifier on the activation across all 160 sensor channels to distinguish the conditions of interest. Classifier performance was determined using leave-one-run-out cross-validation. Data were split into a training dataset, containing the data from 15 runs, and a testing dataset, containing the data from the left-out run. This was done 16 times, using a different testing run each time. The 
250

251

252

253

254

255

256

257

258

259

260

261

262

263

264

265

266

267

268

269

270

271

272

273

analysis was repeated for each point in time, resulting in a decoding accuracy over time. We used the CoSMoMVPA toolbox for MATLAB to conduct our MVPA analyses (Oosterhof et al., 2016).

Cued and distractor orientation: To examine whether there was information about the cued orientation, we trained a classifier to distinguish between the 4 possible cued orientations. These 4 orientations were divided into 2 pairs (see Figure $1 \mathrm{~B}$ ); $22.5^{\circ}$ was paired with $112.5^{\circ}$, and $67.5^{\circ}$ was paired with $157.5^{\circ}$. The classification of orientation was done within each pair separately, and the decoding accuracies were then averaged over pairs. This ensured that the cued orientation was orthogonal to the distractor orientation and comparison orientation. For example, in pair 1 , cued orientation $22.5^{\circ}$ and $112.5^{\circ}$ occurred equally often with each of distractor orientations $67.5^{\circ}$ and $157.5^{\circ}$ (see Figure $1 \mathrm{~B}$ ). Cued and distractor orientations were also orthogonal to the rotation decision, so that only the orientation of the stimulus, and not the participant's decision about it, could drive the classifier. Because the decoding was done within a pair, theoretical chance level was $50 \%$. The decoding analysis for the distractor orientation was the same, as the possible cued and distractor orientations were identical.

Rotation direction: We used coding of the rotation direction as a measure for coding of the decision participants had to make. To determine whether there was information about the rotation direction, we trained a classifier to distinguish between a clockwise and anticlockwise rotation. The rotation direction was orthogonal to both the cued and distractor orientation conditions (e.g., a clockwise decision was equally associated with all 4 possible orientations). There were 2 possible rotation directions, giving a theoretical chance level of $50 \%$. 
274

275

276

277

278

279

280

281

282

283

284

285

286

287

288

289

290

291

292

293

294

295

296

297

298

299

\subsubsection{Statistics}

We used Bayesian statistics to determine the evidence for the coding of information under the null hypothesis (chance decoding) and the alternative hypothesis (above-chance decoding), for each time-point (Dienes, 2011; Jeffreys, 1998; Kass \& Raftery, 1995; Rouder et al., 2009; Wagenmakers, 2007), using the Bayes Factor package in R (Morey \& Rouder, 2018). To test whether the decoding accuracy was above chance, we used a half-Cauchy prior for the alternative, with the default width of 0.707 (Jeffreys, 1998; Rouder et al., 2009; Wetzels et al., 2011). We used a hybrid null that combines a point null centred around chance, with an interval ranging from a standardised effect size of 0 to 0.5 (Morey \& Rouder, 2011). To test whether there was stronger information coding for the cued compared to the distractor orientation, we calculated the difference between decoding accuracies for the cued and distractor orientation. We used a half-Cauchy prior, centred around 0 , with the same width and null interval described above, for the alternative hypothesis to capture directional effects (cued $>$ distractor).

Bayes Factors (BFs) of $<1$ show evidence for the null hypothesis, while BFs of $>1$ show evidence for the alternative hypothesis. BFs between $1 / 3$ and 3 are typically interpreted as showing insufficient evidence, BFs $<1 / 3$ or BFs $>3$ as substantial evidence, and BFs $<1 / 10$ or BFs $>10$ are interpreted as strong evidence (Wetzels et al., 2011). In this study, we defined the onset of strong evidence for above-chance decoding as the second consecutive timepoint with a BF $>10$, and the return to baseline decoding as the second consecutive timepoint with a $B F<1 / 10$.

\section{2. fMRI}

\subsubsection{Participants}

The second part of the study consisted of a behavioural training session and an fMRI session carried out at the MRC Cognition and Brain Sciences Unit (Cambridge, UK). 42 
healthy volunteers participated in the initial training session. Of these, 27 volunteers

301 performed well enough to participate in the fMRI session (at least $90 \%$ accuracy in the final

302 run of the training session). The fMRI data from 3 participants were excluded due to

303 excessive movement, failure to complete the experiment, or low task performance (accuracy

$304<80 \%$ in the fMRI session), resulting in a final fMRI sample of 24 participants (15 female/9

305 male, 23 righthanded $/ 1$ lefthanded, mean age $=27.33$ years, $S D=5.53)$. One of these

306 participants also participated in the MEG experiment and 23 of the participants were new

307 recruits. Participants received $£ 6$ for participation in the training session and $£ 20$ for

308 participation in the $\mathrm{fMRI}$ session, as well as $£ 2.50$ - £3 travel costs per session. All

309 participants provided informed consent, and the study was approved by the Cambridge

310 Psychology Research Ethics Committee.

311

312 2.2.2. Stimuli and experimental procedure

313 fMRI session

314 Participants performed the same task as in the MEG, except for the following 3 changes due

315 to the lower temporal resolution of fMRI. First of all, the order of consecutive trial types was not fully randomised as it was in the MEG version, but was instead counterbalanced within each run, making sure each combination of cued orientation $\mathrm{x}$ distractor orientation $\mathrm{x}$ comparison orientation was equally likely to follow each other combination as well as itself.

319 The order of the rotation direction was balanced separately within each run. Secondly, to keep the order of consecutive trial types intact, error trials were not repeated. Thirdly, participants did not receive trial-wise feedback to avoid feedback of the previous trial influencing the signal of the next trial. Participants still received feedback about their accuracy at the end of each block. Participants completed 8 runs, with 4 blocks per run, and 
same as the MEG experiment, however, the blocks were divided into 8 runs of 4 blocks, instead of 16 runs of 2 blocks. One participant completed 7 instead of 8 runs.

327

328 Training session

329 The training session was the same as described in the MEG experiment section.

330

331

\subsection{3. $f M R I$ acquisition}

332 fMRI scans were acquired using a Siemens 3 Tesla Prisma-Fit scanner (Siemens

333 Healthcare, Erlangen, Germany), with a 32-channel head coil at the MRC Cognition and

334 Brain Sciences Unit, Cambridge, UK. We used a multi-band T2*-weighted echo planar imaging $(\mathrm{EPI})$ acquisition sequence with the following parameters: repetition time $(\mathrm{TR})=$ 1208ms; echo time $(T E)=30 \mathrm{~ms}$; flip angle $=67$ degrees, field of view $=192 \mathrm{~mm}$, multi-band acceleration factor $=2$, no in-plane acceleration, in-plane resolution $=3 \times 3 \mathrm{~mm}, 38$ interleaved slices of $3 \mathrm{~mm}$ slice thickness with 10\% interslice gap. T1-weighted MPRAGE structural images were acquired at the start of the session (resolution $=1 \times 1 \times 1 \mathrm{~mm}$ ). The stimuli were presented on a NNL LCD screen (resolution $=1920 \times 1080$, refresh rate $=60$ $\mathrm{Hz}$ ) using the Psychtoolbox extension in MATLAB (Brainard, 1997; Kleiner et al., 2007; Pelli, 1997). Participants used a 2-button response pad to respond on each trial. To get comfortable with performing the task in the MRI scanner, participants completed 2 practice blocks of the task during the acquisition of the structural scan at the start of the scanning session. Feedback about accuracy was given at the end of each of these practice blocks.

348 The data were pre-processed using SPM 8 (Wellcome Department of Imaging Neuroscience) in MATLAB. EPI images were converted to NIFTII format, spatially realigned 
351

352

353

354

355

356

357

358

359

360

361

362

363

364

365

366

367

368

369

370

371

372

373

374

375

376

SPM12 to allow for multiband acquisitions). Structural scans were co-registered to the mean EPI image and normalised to derive the normalisation parameters needed for the definition of the regions of interest (ROIs).

To obtain activation patterns for the MVPA analysis, we estimated a General Linear Model (GLM) for each participant. There were 16 regressors per run, reflecting a combination of 4 cued orientations $\times 2$ distractor orientations $\times 2$ rotation directions (see Figure 1B). Whole trials were modelled as a single events, lasting from the onset of the first stimulus until the response was given, to account for trial-to-trial variability in response time (Woolgar et al., 2014). Regressors were convolved with the hemodynamic response function of SPM8.

\subsubsection{Regions of interest}

13 frontal and parietal MD ROIs were taken from the parcellated map provided by

Fedorenko et al (2013), which is freely available online at imaging.mrc-

cbu.cam.ac.uk/imaging/MDsystem. The definition of the MD network is activation based, as the map indexes regions that show a univariate increase in activation with increased task demands, across a range of tasks. This is an updated definition of the MD system, with a high degree of overlap with the previous definition, derived from meta-analytic data (Duncan \& Owen, 2000) that we used in previous work (Jackson et al., 2016; Jackson \& Woolgar, 2018; Woolgar, Williams, et al., 2015; Woolgar, Afshar, et al., 2015), and a more recent definition derived from multimodal imaging (Assem et al., 2020). The ROls comprised left and right anterior inferior frontal sulcus (alFS; center of mass $(\mathrm{COM})= \pm 354719$, volume $=$ $5.0 \mathrm{~cm}^{3}$ ), left and right posterior inferior frontal sulcus (pIFS; COM $\pm 403227,5.7 \mathrm{~cm}^{3}$ ), left and right premotor cortex $\left(\mathrm{PM} ; \mathrm{COM} \pm 28-256,9.0 \mathrm{~cm}^{3}\right)$, left and right inferior frontal junction (IFJ; COM $\pm 44432,10.1 \mathrm{~cm}^{3}$ ), left and right anterior insula/frontal operculum ( $\mathrm{Al} / \mathrm{FO} ; \mathrm{COM} \pm 34192,7.9 \mathrm{~cm}^{3}$ ), left and right intraparietal sulcus (IPS; COM $\pm 29-5646$, 
$37734.0 \mathrm{~cm}^{3}$ ), and bilateral anterior cingulate cortex (ACC; COM $01546,18.6 \mathrm{~cm}^{3}$ ). Early visual

378 cortex was defined as area BA 17 (center of mass $=-13,-81,3 / 16,-79,3$, volume $=54$

$379 \mathrm{~cm}^{3}$ ) from the Brodmann template provided with MRIcro (Rorden \& Brett, 2000). ROIs were

380 deformed into native space by applying the inverse of the normalisation parameters for each 381 participant.

382

\subsubsection{Decoding analysis}

We used the same decoding analysis as described in the MEG experiment but in the MEG experiment, we used MVPA to determine what information is present in the pattern of activation for each point in time, whereas for the fMRI data, we determine what information is present in the pattern of activation for each ROI. Within each ROI, we trained an SVM classifier on the betas obtained from the GLM to distinguish the conditions of interest. The classifier performance was determined by using a leave-one-run-out cross-validation. Decoding accuracies were then averaged over hemispheres.

\subsubsection{Statistics}

To test for an effect of attention, MD region, and the interaction between these factors, we 
403

404

405

406

407

408

409

410

411

412

413

414

415

416

417

418

419

420

421

422

423

424

425

426

427

individual MD region and for $\mathrm{V} 1$. We used the same parameters for these tests as described in the MEG section (2.1.6).

\subsection{Model-based MEG-fMRI fusion}

To gain insight into the coding of attended information, unattended information, and decision-related information in both space and time simultaneously, we used model-based MEG-fMRI fusion (Cichy et al., 2014, 2016; Hebart et al., 2018). This method uses

Representational Dissimilarity Matrices (RDMs) (Kriegeskorte et al., 2006, 2008) to abstract away from the imaging modality, allowing us to compare the pattern similarity across neuroimaging modalities. Model-based MEG-fMRI fusion further allows us to determine the match between the representational structure for each timepoint (MEG), and each ROI (fMRI), which can be uniquely explained by a theoretical model (Hebart et al., 2018) (see Figure $6 \mathrm{~A}$ for an overview of this method). We used 3 orthogonal models, coding for the cued orientation, distractor orientation, and rotation direction.

The RDMs consisted of a 16 by 16 matrix comprising each combination of cued orientation (4) $\mathrm{x}$ distractor orientation (2) $\mathrm{x}$ rotation direction (2) (see Figure 1B for an overview of combinations). For each cell in the RDM, we used decoding accuracy as our measure of dissimilarity, with greater decoding accuracy reflecting greater dissimilarity of activation patterns (i.e., distinctiveness of patterns between the conditions). Decoding accuracies were obtained for each cell in the RDM (i.e., pair of trial types) using the same cross-validation procedure as described above and were z-scored per ROI and timepoint. For the MEG data, we constructed an RDM at each timepoint. For each cell in the RDM, we obtained decoding accuracies using a $25 \mathrm{~ms}$ sliding window centred around each timepoint. For the fMRI data, we constructed an RDM for the mean MD and V1 ROls. The mean MD ROI was obtained by calculating the RDM for each individual MD region and averaging 
across regions ${ }^{1}$. We did this separately for each participant, and then averaged the RDMs over participants, resulting in a single RDM per timepoint for MEG, and per ROI for fMRI. We constructed 3 model RDMs: for the cued orientation, distractor orientation, and rotation direction (see Figure 6A). The cued and distractor orientation models coded for: the same orientation (0), a difference of $45^{\circ}(0.5)$, or a difference of $90^{\circ}(1)$; the rotation direction model coded for the same rotation (0) or a different rotation (1). the MEG RDM for each point in time, the fMRI RDM for each ROI, and the model RDM. For each timepoint, ROI, and model, we calculated the difference between two squared semipartial correlation coefficients, using Spearman correlation. Both semi-partial correlation coefficients reflected the proportion of variance shared between MEG and $\mathrm{AMRI}$; one with all models partialled out from the MEG RDM, and the other with all models except the model of interest partialled out from the MEG RDM. By comparing the variance when our model of interest was included or not, we get a measure of the variance shared between MEG and derive a time-course of the model fit for each region.

\subsubsection{Statistics}

446 Because we averaged the RDMs over participants for both MEG and fMRI, we had a single 447 RDM for each timepoint for MEG, and a single RDM for each ROI for fMRI. To estimate the 448 null distribution, we computed 10,000 permutations by shuffling the rows and columns of the 449 group average MEG RDM and ran model-based MEG-fMRI fusion for each ROI using the permuted MEG matrices. For each ROI, we then determined a cluster-inducing threshold for each timepoint by choosing the $95^{\text {th }}$ percentile. We determined the largest maximum cluster

\footnotetext{
1 We could instead have calculated the RDM by extracting the data from one large ROI including all MD regions; in practice this gave a similar RDM so was not considered further.
} 
size in the null distribution. We did this over both ROls to account for multiple comparisons over our 2 ROls. We compared the clusters in our fusion data to the maximum cluster size obtained from the null distribution (equivalent to $p<0.05$, one-tailed, corrected for multiple comparisons for the two ROls and all the timepoints).

456

457

\section{RESULTS}

\subsection{MEG results}

\subsubsection{Behavioural results for MEG session}

460 Participants performed with high accuracy in the MEG session. The mean accuracy was $94.23 \%(S D=3.90)$ before replacing error trials (see Methods), and 99.67\% (SD = 0.56) after replacing error trials where possible, which means very few error trials were included in the analysis ( $0.33 \%$ of trials on average). The mean response time was $667 \mathrm{~ms}$ (SD = $104 \mathrm{~ms})$.

465

\subsubsection{Coding of the cued and distractor orientation over time}

467 To examine the timepoints at which attended visual information was preferentially coded over unattended visual information, we compared the decoding accuracies for the same orientations when presented as the cued or distractor orientation for each point in time. Critically, the coding of the cued and distractor information could not be driven by decisionrelated information, as this was an orthogonal dimension in the design. The decoding accuracies for the cued and distractor orientations are shown in Figure 2. There was strong evidence for above-chance orientation decoding for the cued orientation starting at $85 \mathrm{~ms}$

474 after stimulus onset, which was maintained until after the mean response time. The distractor stimulus orientation could be decoded with a very similar onset to the cued orientation, with strong evidence for above-chance coding from 90ms after stimulus onset.

477 However, unlike the coding of the cued information, coding of the distractor orientation was 
not sustained over time, dropping back to chance within $420 \mathrm{~ms}$ after stimulus onset. The
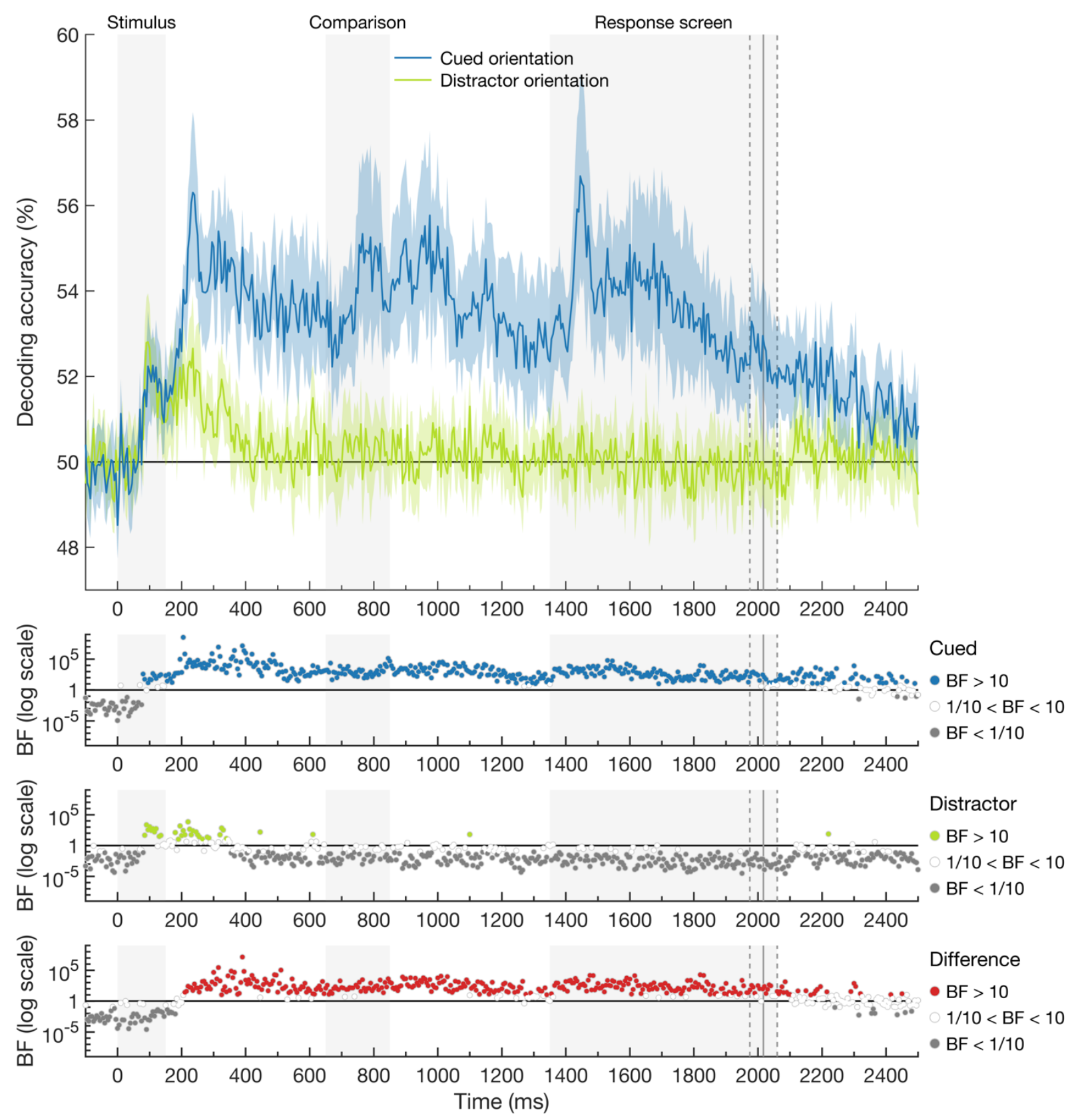

Figure 2. Decoding accuracy of the cued and distractor orientation over time. cued (blue) or not (light green). Theoretical chance is $50 \%$ decoding accuracy, and shaded 
areas around the plot lines show the 95\% confidence intervals. The grey shaded areas show from left to right: 1 ) when the stimulus was on the screen (oriented lines in cued and distractor colours; labelled Stimulus), 2) when the comparison line was on the screen (labelled Comparison), and 3) when the response screen was shown (labelled Response screen). The response screen remained on until participants responded (or $3 s e c s)$. The vertical grey lines show the mean response time with 95\% confidence intervals. Bayes factors are given below the plot on a logarithmic scale for the cued orientation (blue), the distractor orientation (light green) and the difference between the cued and distractor orientation (red). BFs below $1 / 10$ are shown in grey, indicating strong evidence for the null hypothesis. BFs above 10 are shown in the plot colour (blue for the cued orientation, light green for the distractor orientation, and red for the effect of attention), indicating strong evidence for the alternative hypothesis. BF between $1 / 10$ and 10 are shown in white. There was strong evidence for coding of the cued orientation from $85 \mathrm{~ms}$ after the onset of the stimulus which was maintained until after the response was given. There was strong evidence for coding of the distractor orientation from $90 \mathrm{~ms}$ after the onset of the stimulus, but this was not sustained over time. There was strong evidence for an effect of attention from $215 \mathrm{~ms}$ after the onset of the stimulus until after the mean response time.

505

\subsubsection{Coding of the rotation direction over time}

507 To examine the time-course of decision-related information coding, we determined the coding of the rotation direction over time by training a linear classifier to distinguish between clockwise and anti-clockwise rotations. Participants had to actively manipulate the cued orientation in combination with the comparison orientation to determine the rotation. The

511 decoding of the rotation direction over time is shown in Figure 3. We observed above-

512 chance decoding of the rotation direction from $170 \mathrm{~ms}$ after the onset of the comparison line,

$513820 \mathrm{~ms}$ after the onset of the attended and unattended orientations, which was maintained

514 until after the mean response time. 


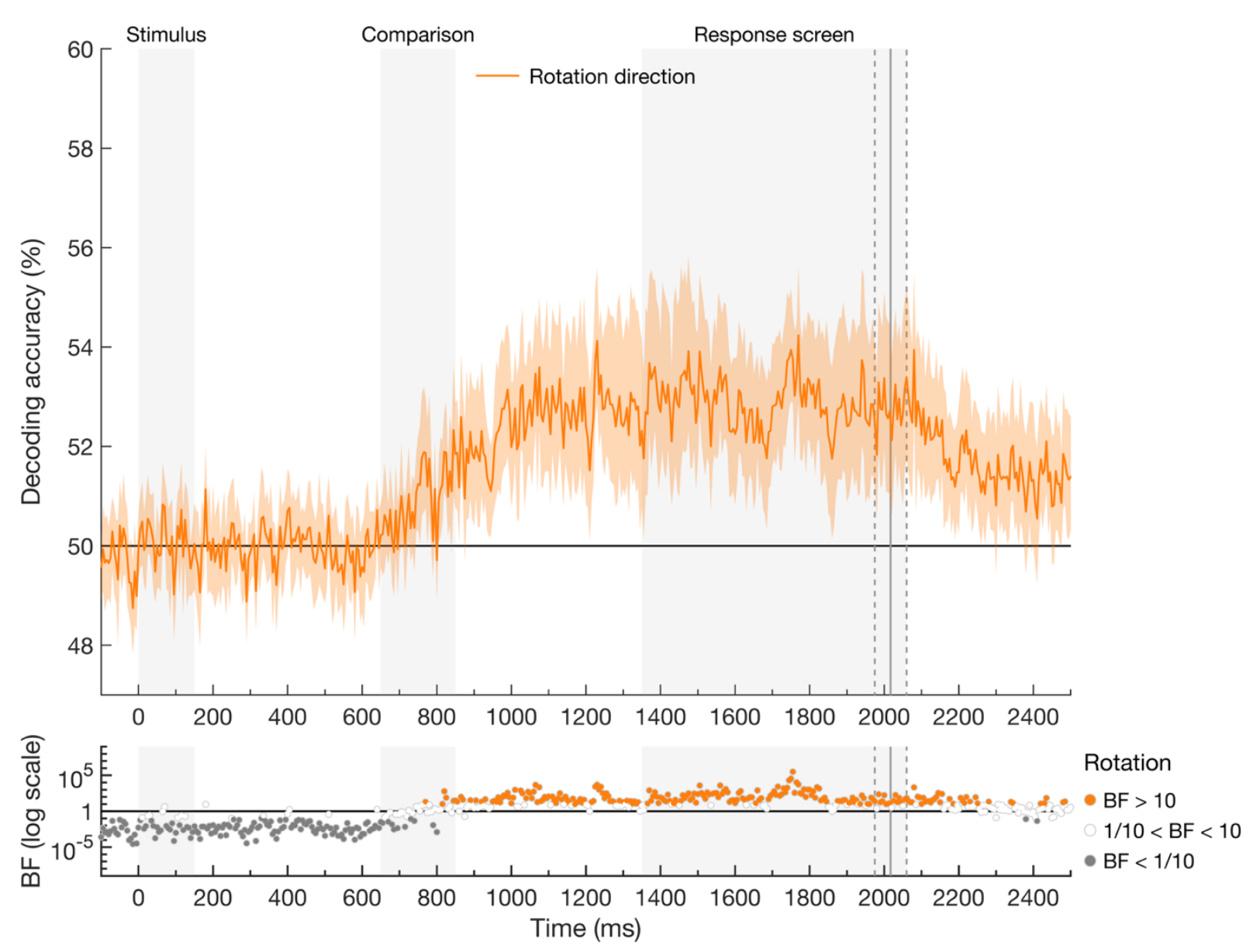

Figure 3. Decoding accuracy of the rotation direction over time. Classifiers were trained

518 to discriminate the rotation direction, which could be clockwise or anti-clockwise. The

519 rotation direction, about which participants had to make a decision, could be decoded from

$520170 \mathrm{~ms}$ after the onset of the comparison line (820ms after stimulus onset) and was

521 maintained until after the mean response time. Plotting conventions are as in Figure 2.

522

523

\section{2. fMRI results}

524

\subsubsection{Behavioural results for $\mathrm{fMRI}$ session}

525

Participants performed the task in the fMRI session with high accuracy (mean accuracy =

$94.45 \%$, SD $=4.91)$. Included participants all had a mean accuracy above $80 \%$. The mean

response time was $658 \mathrm{~ms}(\mathrm{SD}=140 \mathrm{~ms})$ from response screen onset.

530 To examine whether the effect of attention observed in the MEG data pertained to V1 and

531 the MD regions, we decoded the cued and distractor orientations for these ROIs. We were 
532

533

534

535

536

537

538

539

540

541

542

543

544

545

546

547

548

549

550

551

552

553

554

555

556

able to separate these conditions due to the orthogonal design. The decoding accuracies for the cued and distractor orientations, averaged across the MD regions and in $\mathrm{V} 1$, are shown in Figure 4A. We used a Bayesian ANOVA with attention (orientation coding of cued vs. distractor stimuli) and MD region (alFS, pIFS, PM, IFJ, AI/FO, IPS, and ACC; data collapsed across hemispheres) as within-subject factors. We found a main effect of attention (BF > $100)$, but there was not enough evidence for a main effect of region $(B F=0.44)$. There was evidence for an interaction between attention and region $(B F=9.60)$ justifying a further analysis of the effect of attention in individual MD regions (see Figure 4B). There was strong evidence for an effect of attention (cued > distractor coding) in alFS, pIFS, PM, IFJ, and IPS, and some evidence for an effect of attention in Al/FO and ACC (see Figure 4B).

To determine whether the orientation coding for the cued and distractor orientations was above chance, we performed Bayesian t-tests for the mean MD regions as well as the individual MD regions. There was strong evidence for above-chance coding for the cued orientation in the mean $\mathrm{MD}$ regions (mean accuracy $=55.62 \%, \mathrm{BF}>100$ ), while decoding of the distractor orientation was at chance (mean accuracy $=49.56 \%, \mathrm{BF}<0.01)$. There was above-chance decoding for the cued orientation in all of the MD regions. Strong evidence for chance level decoding for the distractor orientation was found for alFS, pIFS, PM, IFJ, Al/FO, and IPS. There was some evidence for chance decoding for the distractor orientation in ACC, and the 95\% confidence intervals for this region overlapped with chance.

We also found an effect of attention in V1 (BF > 100), with stronger coding for the cued orientations compared to the distractor orientations. V1 coded for the cued orientation (mean accuracy $=66.34 \%, B F>100$ ), while there was no evidence for coding of the identical orientations when they were distractors. There was some evidence for chance decoding of the distractor orientation in V1 (mean accuracy $=51.29 \%, \mathrm{BF}=0.28$ ), and the $95 \%$ confidence intervals overlapped with chance. 

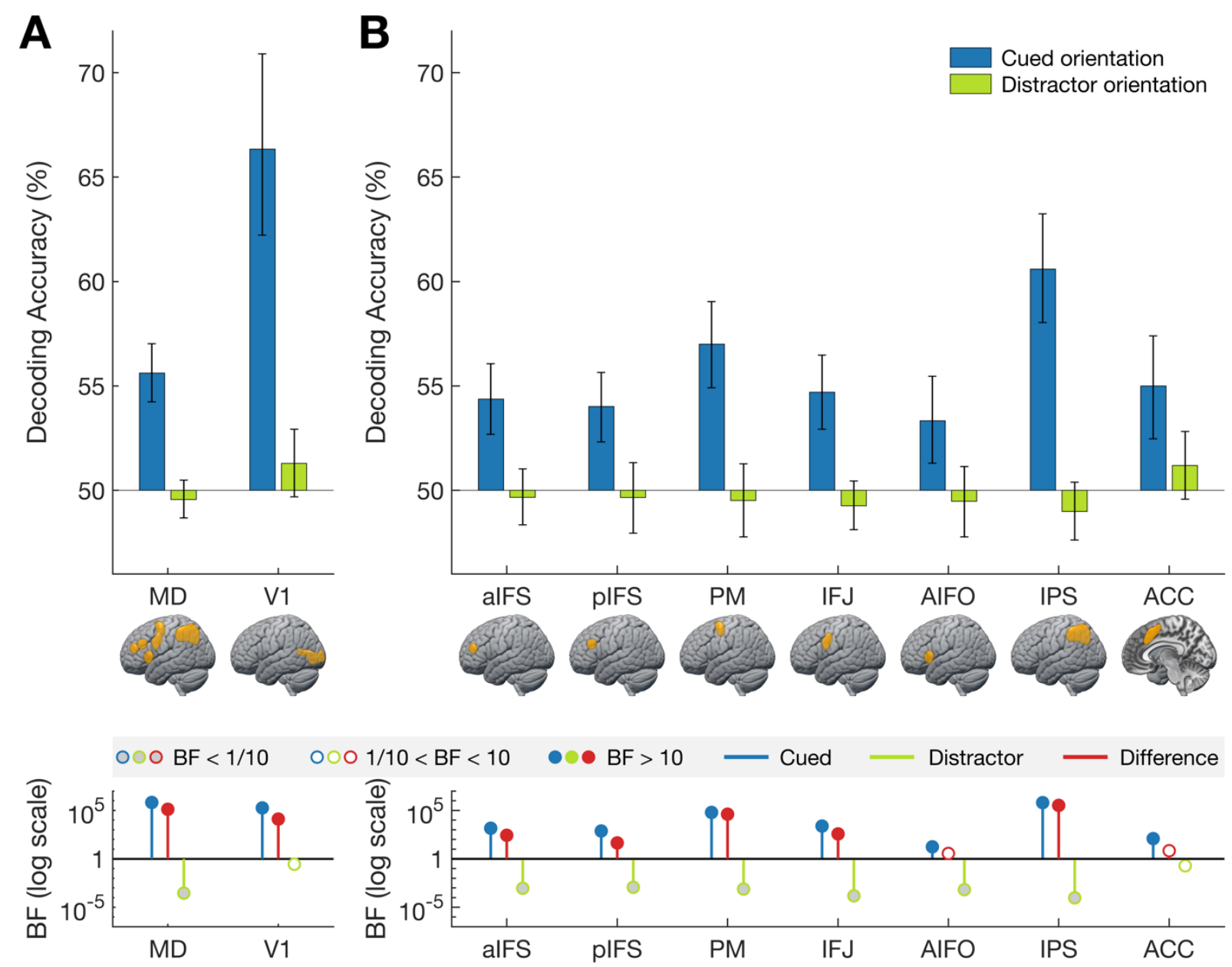

Figure 4. Decoding accuracy of the cued and distractor orientation $(A)$ averaged across the $M D$ regions and in $V 1$; and $(B)$ in individual $M D$ regions. In both $A$ and $B$, classifiers were trained to discriminate stimulus orientation when the orientation was either cued (blue) or not (light green). Theoretical chance is 50\%, and error bars indicate $95 \%$ confidence intervals. The Bayes factors for the decoding accuracies are shown below the plot in blue (cued orientation) and light green (distractor orientation), and the BFs for the difference (cued>distractor coding) are shown in red. All BFs are shown on a logarithmic scale. BFs $>10$ are marked in the plot colour, BFs $<1 / 10$ are marked in grey, and BFs in between this range are marked in white. In all of the MD regions, and in V1, there was strong evidence for coding of the cued orientation, but not for the distractor orientation. There was strong evidence for an effect of attention for most of the MD regions (alFS, pIFS, PM, IFJ, Al/FO, and IPS) as well as average MD and V1.

\subsubsection{Coding of the rotation direction in the MD regions and V1}

573 To examine whether there was information about the rotation direction, which is our indicator

574 of the decision, in the MD regions and V1, we trained a linear classifier to distinguish

575 between a clockwise and anti-clockwise rotation direction. Since error trials were included in

576 the analysis to maintain the orthogonality of the different features, the decoded rotation 
577 direction on these trials does not always reflect the decision of the participant. However, as

578 this only occurred on $\sim 6 \%$ of trials, the predominant influence will be the decision. Figure $5 \mathrm{~A}$

579 shows the decoding accuracies for the rotation direction in the MD regions and V1. We used

580 Bayesian t-tests to determine the evidence for chance versus above-chance decoding. We

581 observed strong evidence for above-chance coding of rotation direction across the MD

582 regions on average (mean accuracy $=52.43 \%, B F=45.89$ ), and in PM and IPS individually

583 (Figure 5B). Although the average decoding accuracy found in V1 was similar to the MD

584 regions, greater variance in V1 was reflected in an inconclusive BF (mean accuracy =

$58552.67 \%, B F=1.85)$. There was substantial, but not strong, evidence for chance decoding in

586 alFS, pIFS, and IFJ, and insufficient evidence for rotation direction coding in the other MD

587 regions.

588
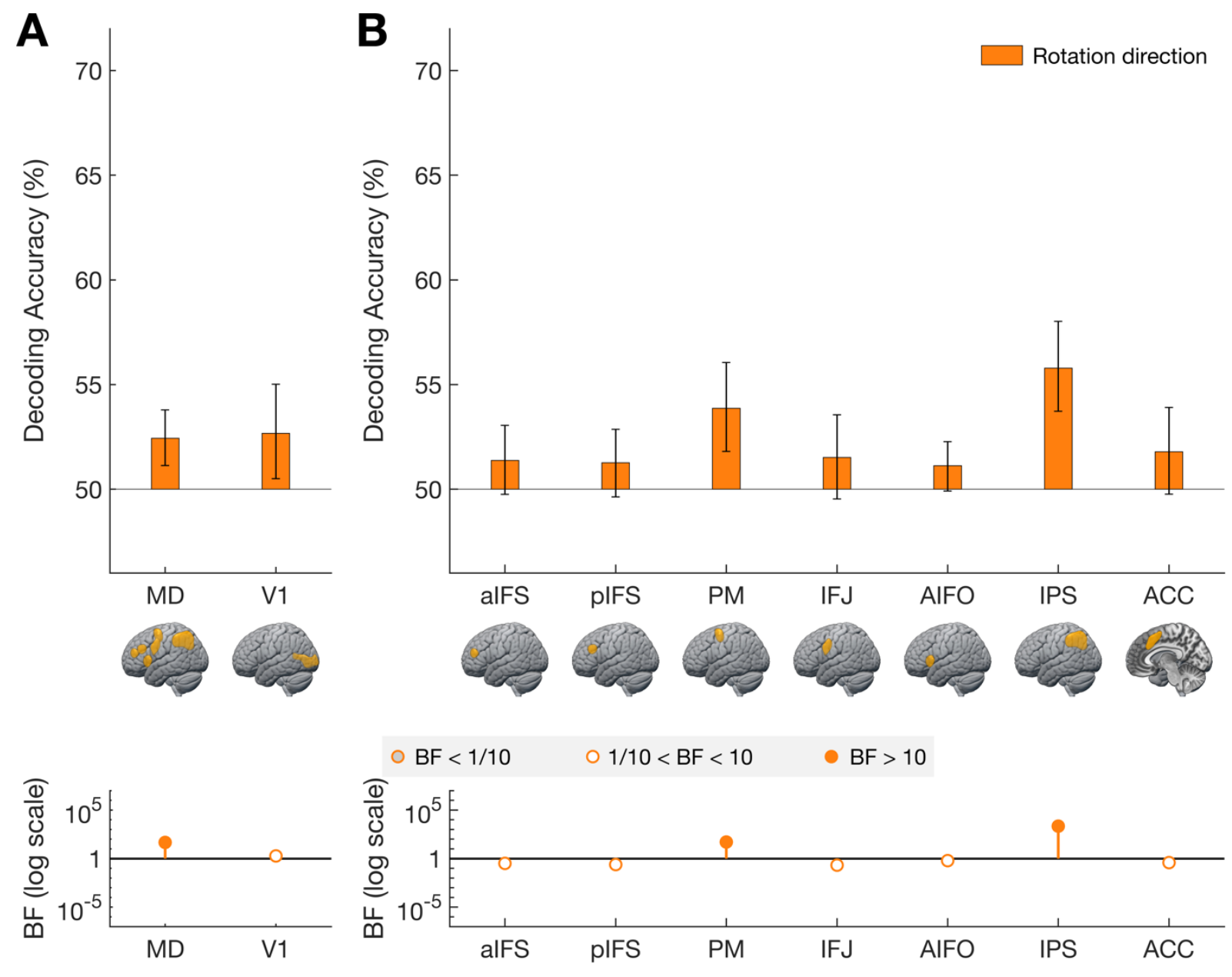

after the mean response time (Figure 6C).

614

\subsubsection{Distractor orientation}

617 region or $\mathrm{V} 1$. rotation direction coding in $\mathrm{V} 1$.

\subsection{Model-based MEG-fMRI fusion results} rotation direction.

\subsubsection{Cued orientation}

Figure 5. Decoding accuracy of the rotation direction (decision) (A) averaged across the $M D$ regions and in $V 1$ and $(B)$ in individual $M D$ regions. In both $A$ and $B$, classifiers were trained to discriminate the rotation direction, which could be clockwise or anticlockwise. Plotting conventions are as in Figure 4. The rotation direction could be decoded from the MD regions (average, PM and IPS), while there was insufficient information for

We used model-based MEG-fMRI fusion to investigate whether the MD regions code attended information before a decision is made (see Figure $6 \mathrm{~A}$ ). Figure $6 \mathrm{~B}$ and $6 \mathrm{C}$ show the commonality coefficient over time for our 2 ROls: the MD regions and $\mathrm{V} 1$. The commonality refers to the part of the variance that is shared between the MEG and fMRI RDMs, which can be uniquely explained by our models for the cued orientation, distractor orientation, and

In the MD regions, there was a significant cluster of commonality for the cued orientation from 555ms until $640 \mathrm{~ms}$ after the onset of the stimulus (Figure 6B). Critically, this cluster occurred before the onset of the comparison line, and therefore before participants could make the decision. A second cluster started at $1140 \mathrm{~ms}, 490 \mathrm{~ms}$ after the onset of the comparison line and was maintained until after the average response time. In V1, cued orientation commonality coefficients were significant from $225 \mathrm{~ms}$ until $505 \mathrm{~ms}$ after the onset of the stimulus, and again from $1210 \mathrm{~ms}, 560 \mathrm{~ms}$ after the onset of the comparison line, until 


\subsubsection{Rotation direction}

620 We observed a cluster of significant commonality for the rotation direction, which is an index

621 of the decision participants had to make, in the MD regions starting from $1140 \mathrm{~ms}, 490 \mathrm{~ms}$

622 after the onset of the comparison line, which lasted until after the participant responded. V1

623 showed a similar pattern over time, with a significant rotation direction cluster from 1160ms,

$624510 m s$ after comparison line onset.

625
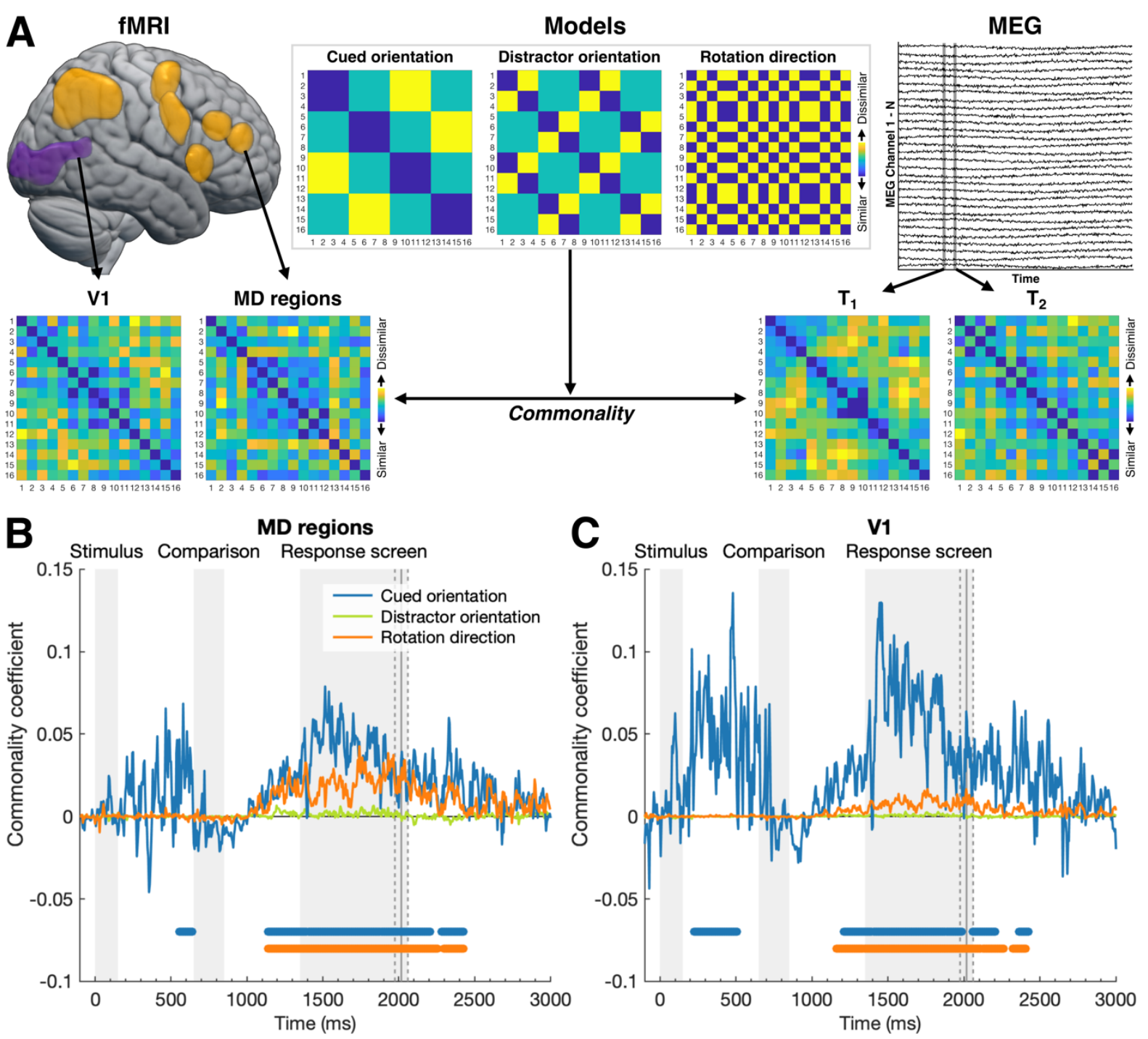

Figure 6. Model-based MEG - fMRI fusion methods (A) and results over time for the $M D$ regions $(B)$ and $\boldsymbol{V 1}(\boldsymbol{C})$. A gives an overview of the model-based $M E G-f M R I$ fusion method. We used RDMs to abstract away from the imaging modality. Each cell in the RDM corresponds to the dissimilarity between 2 specific trial types. A trial type is a combination of the cued orientation, distractor orientation, and rotation direction. Trial types 1-16 in the RDMs correspond to the 16 trial types listed in Figure 1B. We created an RDM for each region of interest (V1 and mean MD regions) for the $\mathrm{MRI}$ data, for each time-point in the 
634

635

636

637

638

639

640

641

642

643

644

645

646

647

648

649

650

651

652

653

654

655

656

657

658

659

660

661

662

663

664

MEG data, and for the 3 models (cued orientation, distractor orientation, and rotation direction). We then determined commonality: the part of the variance that is shared between the MEG RDM for each timepoint and the fMRI RDM for each ROI, that can be uniquely explained by each theoretical model. This results in a time-course of commonality coefficients for each ROI and model. $\mathbf{B}$ and $\mathbf{C}$ show the time-course of commonality for the $\mathrm{MD}$ regions and $\mathrm{V} 1$ respectively. The different lines depict the commonality explained by the model for the cued orientation (blue), the distractor orientation (light green), and the rotation direction (orange). Significant timepoints (equivalent to $p<0.05$, one-tailed) were obtained with a cluster-corrected randomisation test, corrected for multiple comparisons across ROIs and timepoints, and are shown at the bottom of the plots. Plotting conventions are as in Figure 2 and 3 except that the dots in the bottom of this plot show significant time-points, not Bayes Factors.

\section{DISCUSSION}

A wealth of $\mathrm{fMRI}, \mathrm{MEG}$ and non-human primate studies have shown preferential coding of cued compared to distractor information (Bode \& Haynes, 2009; Goddard et al., 2019; Harel et al., 2014; Jackson et al., 2016; Li et al., 2007; Rao et al., 1997; Sigala et al., 2008; Stiers et al., 2010; Stokes et al., 2013; Waskom et al., 2014; Woolgar, Afshar, et al., 2015; Woolgar, Hampshire, et al., 2011; Woolgar, Thompson, et al., 2011; Woolgar, Williams, et al., 2015). However, many paradigms manipulate attention by asking participants to respond to the cued (but not the distractor) stimulus, which means they cannot distinguish between preferential coding of cued information due to attentional selection and maintenance of relevant information, and coding due to decision-making processes performed on the attended information. We used MEG, fMRI, and model-based MEG-fMRI fusion to distinguish between these explanations. Our MEG data showed that attention affects stimulus processing around $215 \mathrm{~ms}$ after stimulus onset, before participants had the necessary information to make a decision. Then, when the comparison line was presented, the decision about the rotation direction could be decoded approximately $170 \mathrm{~ms}$ later. Our $\mathrm{fMRI}$ data showed the MD regions coded attended visual information, and did so more strongly than distracting visual information, even though the decision to be made was orthogonal to the stimulus itself. The orthogonal decision representation was also present in 
665

666

667

668

669

670

671

672

673

674

675

676

677

678

679

680

681

682

683

684

685

686

687

688

689

690

the MD system, specifically in PM and IPS. We also found an effect of attention in V1, with stronger coding of the cued compared to distractor orientations, in line with previous studies (Jackson et al., 2016; Jehee et al., 2012; Pratte et al., 2013; Woolgar, Hampshire, et al., 2011). Our model-based MEG-fMRI fusion analysis showed evidence for the coding of attended orientation information in the MD regions before participants could make a decision. These data are consistent with the interpretation of previous results as effects of attention on the processing of visual stimuli, with a stronger coding of attended compared to unattended visual information in the brain. This could be driven by an enhancement of the attended visual information, a suppression of the unattended visual information, or both. The finding that the MD regions preferentially code for attended information over unattended information, even when the decision is orthogonal, is in line with the finding of Hon and colleagues (2006), who used univariate analyses to show that the average MD response reflected changes in cued information when no decision was needed, or when the decision was unrelated to the cued information. Our results take the inference beyond simple activation changes and confirm that decision-making processes are not required for the prioritisation of cued over distractor information.

Our results also show that, in addition to coding the attended information, MD regions also code for decision-related information. The PM and IPS, which are part of the MD network, held information about the rotation direction, which participants had to make a decision about. The time-course with which this information emerged, from about 170ms after the onset of the comparison line, fits well with a previous study which found a perceptual decision on a pure noise image could be decoded around 140-180ms (Bode et al., 2012). The fast time-course suggests the coding of rotation direction information could reflect the accumulation of decision-related information, before the participant is able to respond. This is in line with the description of decision-making as the accumulation of evidence towards one of two decision alternatives until a decision boundary is reached (Gold 

comment on the exact delay between the onset of decision information coding and the response, as our task involved a delayed response - participants could only respond after the response screen came on. to the literature by combining neuroimaging techniques, giving us resolution in both space and time for a single paradigm. Our MEG data show that the effect of attention emerges before participants have all of the information needed to make a decision, while our fMRI data show that the MD regions code both attended visual information and the decision participants have to make about the stimulus. Moreover, we formally related the two results using model-based MEG-fMRI fusion in order to estimate the time-course of response in the MD network and V1 separately. The data suggest that visual information arises first in V1, but that both $\mathrm{V} 1$ and the MD regions code for information about the attended orientation before participants can begin to make a decision. Orthogonal decision-related signals in MD cortex arose only after the comparison line was presented. The MEG-fMRI fusion method is an exciting step towards resolving neural processes in both time and space, but is limited in sensitivity since it is only able to pick up effects that are present in both the MEG data and the fMRI data (see Cichy \& Oliva (2020) for an overview of possible limitations). For instance, although our MEG data revealed transient coding of unattended information, this was not evident in the fusion results, presumably because the much slower time resolution of $\mathrm{fMRI}$ obscured its detection. This highlights the utility of considering the data from each neuroimaging technique separately, as well as together.

714 literature in non-human primates showing the same neural populations can simultaneously 
717 prefrontal cortex can maintain information about relevant and irrelevant sensory information,

718 as well as the saccade response, over the course of a trial. Our study also provides

719 evidence for a multi-faceted response of the MD regions, although we cannot determine

720 whether this is driven by the same population of neurons within those brain areas. The

721 flexible MD response observed in our study, reflecting relevant visual information as well as

722 decisions about this information, is consistent with the proposal that these regions play a key

723 role in associating different types of information by integrating information over multiple

724 sources (Duncan, et al., 2020). The MD system is well placed to achieve this, being widely distributed and strongly interconnected across the cortex (Assem et al., 2020; Duncan et al., 2020). In addition, our results emphasise an explicit role of the MD regions in selective attention, with preferential coding of the information that is task relevant separately from functional decisions.

In summary, our MEG results show that attention affects the representation of visual stimuli in advance of decision-making processes, while the fMRI results show that the MD regions code for attended visual information as well as decisions about this information. The MEG-fMRI fusion results show the spatio-temporal unfolding of these processes, suggesting that the MD regions represent information about the attended stimulus before participants can begin to make a decision. These results emphasise a key role for the MD regions in selective attention and are consistent with the proposal that these regions integrate different types of information for decision-making. Our multimodal data demonstrate that selective attention and decision-making have separable bases in neural coding.

738

\section{ACKNOWLEDGEMENTS}

740 This work was supported by the Australian Research Council (ARC) Centre of Excellence in

741 Cognition and its Disorders (CE110001021), International Research Training Program

742 Scholarships from Macquarie University awarded to DM, an ARC Discovery Project 
743 (DP170101840) awarded to ANR and AW, and by the Medical Research Council (UK)

744 intramural funding (SUAG/052/G101400) awarded to AW.

745

746

\section{REFERENCES}

747

Aoi, M. C., Mante, V., \& Pillow, J. W. (2020). Prefrontal cortex exhibits multidimensional dynamic encoding during decision-making. Nature Neuroscience, 23(11), 14101420. https://doi.org/10.1038/s41593-020-0696-5

750

Assem, M., Glasser, M. F., Van Essen, D. C., \& Duncan, J. (2020). A Domain-General Cognitive Core Defined in Multimodally Parcellated Human Cortex. Cerebral Cortex, 30(8), 4361-4380. https://doi.org/10.1093/cercor/bhaa023

Battistoni, E., Kaiser, D., Hickey, C., \& Peelen, M. V. (2018). Spatial attention follows category-based attention during naturalistic visual search: evidence from MEG decoding. BioRxiv, 390807. https://doi.org/10.1101/390807

Bode, S., \& Haynes, J.-D. (2009). Decoding sequential stages of task preparation in the 757 human brain. Neurolmage, 45(2), 606-613. https://doi.org/10.1016/j.neuroimage.2008.11.031

Bode, S., Sewell, D. K., Lilburn, S., Forte, J. D., Smith, P. L., \& Stahl, J. (2012). Predicting 760 Perceptual Decision Biases from Early Brain Activity. Journal of Neuroscience, 32(36), 12488-12498. https://doi.org/10.1523/JNEUROSCI.1708-12.2012

Brainard, D. H. (1997). The Psychophysics Toolbox. Spatial Vision, 10(4), 433-436. https://doi.org/10.1163/156856897X00357

Carrasco, M. (2011). Visual attention: The past 25 years. Vision Research, 51(13), 1484765 1525. https://doi.org/10.1016/j.visres.2011.04.012 
769 Cichy, Radoslaw Martin, Pantazis, D., \& Oliva, A. (2014). Resolving human object recognition in space and time. Nature Neuroscience, 17(3), 455-462. https://doi.org/10.1038/nn.3635

Cichy, Radoslaw Martin, Pantazis, D., \& Oliva, A. (2016). Similarity-Based Fusion of MEG and fMRI Reveals Spatio-Temporal Dynamics in Human Cortex During Visual Object Recognition. Cerebral Cortex, 26(8), 3563-3579. https://doi.org/10.1093/cercor/bhw135

Desimone, R., \& Duncan, J. (1995). Neural Mechanisms of Selective Visual Attention. Annual Review of Neuroscience, 18(1), 193-222. https://doi.org/10.1146/annurev.ne.18.030195.001205

Dienes, Z. (2011). Bayesian Versus Orthodox Statistics: Which Side Are You On? Perspectives on Psychological Science, 6(3), 274-290. https://doi.org/10.1177/1745691611406920

Dosenbach, N. U. F., Visscher, K. M., Palmer, E. D., Miezin, F. M., Wenger, K. K., Kang, H. https://doi.org/10.1016/j.neuron.2006.04.031

Duncan, J. (2001). An adaptive coding model of neural function in prefrontal cortex. Nature Reviews Neuroscience, 2(11), 820-829. https://doi.org/10.1038/35097575 for intelligent behaviour. Trends in Cognitive Sciences, 14(4), 172-179. https://doi.org/10.1016/j.tics.2010.01.004 
794

795

796

797

798

799

800

801

802

803

804

805

806

807

808

809

810

811

812

813

814

815

816

817

818

819

Duncan, J., \& Owen, A. M. (2000). Common regions of the human frontal lobe recruited by diverse cognitive demands. Trends in Neurosciences, 23(10), 475-483. https://doi.org/10.1016/S0166-2236(00)01633-7

Fedorenko, E., Duncan, J., \& Kanwisher, N. (2013). Broad domain generality in focal regions of frontal and parietal cortex. Proceedings of the National Academy of Sciences, 110(41), 16616-16621. https://doi.org/10.1073/pnas.1315235110

Fox, M. D., Snyder, A. Z., Vincent, J. L., Corbetta, M., Essen, D. C. V., \& Raichle, M. E. (2005). The human brain is intrinsically organized into dynamic, anticorrelated functional networks. Proceedings of the National Academy of Sciences, 102(27), 9673-9678. https://doi.org/10.1073/pnas.0504136102

Fusi, S., Miller, E. K., \& Rigotti, M. (2016). Why neurons mix: high dimensionality for higher cognition. Current Opinion in Neurobiology, 37, 66-74. https://doi.org/10.1016/j.conb.2016.01.010

Goddard, E., Carlson, T. A., \& Woolgar, A. (2019). Spatial and feature-selective attention have distinct effects on population-level tuning. BioRxiv, 530352. https://doi.org/10.1101/530352

Gold, J. I., \& Shadlen, M. N. (2007). The Neural Basis of Decision Making. Annual Review of Neuroscience, 30(1), 535-574. https://doi.org/10.1146/annurev.neuro.29.051605.113038

Grootswagers, T., Wardle, S. G., \& Carlson, T. A. (2016). Decoding Dynamic Brain Patterns from Evoked Responses: A Tutorial on Multivariate Pattern Analysis Applied to Time Series Neuroimaging Data. Journal of Cognitive Neuroscience, 29(4), 677-697. https://doi.org/10.1162/jocn_a_01068

Harel, A., Kravitz, D. J., \& Baker, C. I. (2014). Task context impacts visual object processing differentially across the cortex. Proceedings of the National Academy of Sciences, 111(10), E962-E971. https://doi.org/10.1073/pnas.1312567111 
820

821

822

823

824

825

826

827

828

829

830

831

832

833

834

835

836

837

838

839

840

841

842

843

844

Haynes, J.-D. (2015). A Primer on Pattern-Based Approaches to fMRI: Principles, Pitfalls, and Perspectives. Neuron, 87(2), 257-270. https://doi.org/10.1016/j.neuron.2015.05.025

Hebart, M. N., \& Baker, C. I. (2017). Deconstructing multivariate decoding for the study of brain function. Neurolmage. https://doi.org/10.1016/j.neuroimage.2017.08.005

Hebart, M. N., Bankson, B. B., Harel, A., Baker, C. I., \& Cichy, R. M. (2018). The representational dynamics of task and object processing in humans. Elife, 7, e32816.

Heekeren, H. R., Marrett, S., \& Ungerleider, L. G. (2008). The neural systems that mediate human perceptual decision making. Nature Reviews Neuroscience, 9(6), 467-479. https://doi.org/10.1038/nrn2374

Hon, N., Epstein, R. A., Owen, A. M., \& Duncan, J. (2006). Frontoparietal Activity with Minimal Decision and Control. Journal of Neuroscience, 26(38), 9805-9809. https://doi.org/10.1523/JNEUROSCI.3165-06.2006

Jackson, J. B., Feredoes, E., Rich, A. N., Lindner, M., \& Woolgar, A. (2020). Concurrent neuroimaging and neurostimulation reveals a causal role for dIPFC in coding of taskrelevant information. BioRxiv, 2020.04.22.054742. https://doi.org/10.1101/2020.04.22.054742

Jackson, J., Rich, A. N., Williams, M. A., \& Woolgar, A. (2016). Feature-selective Attention in Frontoparietal Cortex: Multivoxel Codes Adjust to Prioritize Task-relevant Information. Journal of Cognitive Neuroscience, 29(2), 310-321. https://doi.org/10.1162/jocn_a_01039

Jackson, J., \& Woolgar, A. (2018). Adaptive coding in the human brain: Distinct object features are encoded by overlapping voxels in frontoparietal cortex. Cortex. https://doi.org/10.1016/j.cortex.2018.07.006

Jeffreys, H. (1998). The Theory of Probability. OUP Oxford. 
845 Jehee, J. F. M., Ling, S., Swisher, J. D., Bergen, R. S. van, \& Tong, F. (2012). Perceptual

$846 \quad$ Learning Selectively Refines Orientation Representations in Early Visual Cortex.

$847 \quad$ Journal of Neuroscience, 32(47), 16747-16753.

$848 \quad$ https://doi.org/10.1523/JNEUROSCI.6112-11.2012

849 Kadohisa, M., Petrov, P., Stokes, M., Sigala, N., Buckley, M., Gaffan, D., Kusunoki, M., \&

850 Duncan, J. (2013). Dynamic Construction of a Coherent Attentional State in a

$851 \quad$ Prefrontal Cell Population. Neuron, 80(1), 235-246.

852 https://doi.org/10.1016/j.neuron.2013.07.041

853 Kass, R. E., \& Raftery, A. E. (1995). Bayes Factors. Journal of the American Statistical Association, 90(430), 773-795. https://doi.org/10.1080/01621459.1995.10476572

855

Kleiner, M., Brainard, D., \& Pelli, D. (2007). What's new in Psychtoolbox-3? Perception 36

856 ECVP Abstract Supplement.

857 Kriegeskorte, N., Goebel, R., \& Bandettini, P. (2006). Information-based functional brain

858 mapping. Proceedings of the National Academy of Sciences, 103(10), 3863-3868.

859 https://doi.org/10.1073/pnas.0600244103

860

Kriegeskorte, N., Mur, M., \& Bandettini, P. A. (2008). Representational similarity analysis -

861 connecting the branches of systems neuroscience. Frontiers in Systems

862 Neuroscience, 2. https://doi.org/10.3389/neuro.06.004.2008

863

Li, S., Ostwald, D., Giese, M., \& Kourtzi, Z. (2007). Flexible Coding for Categorical Decisions in the Human Brain. Journal of Neuroscience, 27(45), 12321-12330. https://doi.org/10.1523/JNEUROSCI.3795-07.2007

866

Löffler, A., Haggard, P., \& Bode, S. (2019). Decoding Changes of Mind in Voluntary Action867 Dynamics of Intentional Choice Representations. Cerebral Cortex. 
869 Morey, R. D., \& Rouder, J. N. (2011). Bayes factor approaches for testing interval null

870 hypotheses. Psychological Methods, 16(4), 406-419.

871 https://doi.org/10.1037/a0024377

872 Morey, R. D., \& Rouder, J. N. (2018). BayesFactor: Computation of Bayes Factors for Common Designs. https://CRAN.R-project.org/package=BayesFactor

874 Oostenveld, R., Fries, P., Maris, E., \& Schoffelen, J.-M. (2011). FieldTrip: Open Source

875 Software for Advanced Analysis of MEG, EEG, and Invasive Electrophysiological

876 Data. Computational Intelligence and Neuroscience, 2011, 1-9.

877 https://doi.org/10.1155/2011/156869

878

Oosterhof, N. N., Connolly, A. C., \& Haxby, J. V. (2016). CoSMoMVPA: Multi-Modal

879 Multivariate Pattern Analysis of Neuroimaging Data in Matlab/GNU Octave. Frontiers

880 in Neuroinformatics, 10. https://doi.org/10.3389/fninf.2016.00027

881

Pelli, D. G. (1997). The VideoToolbox software for visual psychophysics: Transforming

882 numbers into movies. Spatial Vision, 10(4), 437-442.

883

Pestilli, F., \& Carrasco, M. (2005). Attention enhances contrast sensitivity at cued and impairs it at uncued locations. Vision Research, 45(14), 1867-1875.

885 https://doi.org/10.1016/j.visres.2005.01.019

Posner, M. I. (1980). Orienting of attention. Quarterly Journal of Experimental Psychology, 32(1), 3-25. https://doi.org/10.1080/00335558008248231

888

Pratte, M. S., Ling, S., Swisher, J. D., \& Tong, F. (2013). How attention extracts objects from 889 noise. Journal of Neurophysiology, 110(6), 1346-1356.

890 https://doi.org/10.1152/jn.00127.2013

891 Rao, S. C., Rainer, G., \& Miller, E. K. (1997). Integration of What and Where in the Primate 892 Prefrontal Cortex. Science, 276(5313), 821-824. 
894 Ratcliff, R., \& McKoon, G. (2007). The Diffusion Decision Model: Theory and Data for Two895 Choice Decision Tasks. Neural Computation, 20(4), 873-922.

896 https://doi.org/10.1162/neco.2008.12-06-420

897 Rens, N., Bode, S., Burianová, H., \& Cunnington, R. (2017). Proactive Recruitment of 898 Frontoparietal and Salience Networks for Voluntary Decisions. Frontiers in Human Neuroscience, 11. https://doi.org/10.3389/fnhum.2017.00610

900 Rigotti, M., Barak, O., Warden, M. R., Wang, X.-J., Daw, N. D., Miller, E. K., \& Fusi, S. 901 (2013). The importance of mixed selectivity in complex cognitive tasks. Nature,

902 497(7451), 585-590. https://doi.org/10.1038/nature12160

903

Rorden, C., \& Brett, M. (2000). Stereotaxic Display of Brain Lesions [Research article]. Behavioural Neurology. https://doi.org/10.1155/2000/421719

Rouder, J. N., Morey, R. D., Speckman, P. L., \& Province, J. M. (2012). Default Bayes factors for ANOVA designs. Journal of Mathematical Psychology, 56(5), 356-374. https://doi.org/10.1016/j.jmp.2012.08.001

Rouder, J. N., Speckman, P. L., Sun, D., Morey, R. D., \& Iverson, G. (2009). Bayesian t tests for accepting and rejecting the null hypothesis. Psychonomic Bulletin \& Review, 16(2), 225-237. https://doi.org/10.3758/PBR.16.2.225

911 Sàenz, M., Buraĉas, G. T., \& Boynton, G. M. (2003). Global feature-based attention for motion and color. Vision Research, 43(6), 629-637. https://doi.org/10.1016/S00426989(02)00595-3

914 Seeley, W. W., Menon, V., Schatzberg, A. F., Keller, J., Glover, G. H., Kenna, H., Reiss, A. L., \& Greicius, M. D. (2007). Dissociable Intrinsic Connectivity Networks for Salience Processing and Executive Control. Journal of Neuroscience, 27(9), 2349-2356. https://doi.org/10.1523/JNEUROSCI.5587-06.2007

918 Sigala, N., Kusunoki, M., Nimmo-Smith, I., Gaffan, D., \& Duncan, J. (2008). Hierarchical coding for sequential task events in the monkey prefrontal cortex. Proceedings of the 
920

921

922

923

924

925

926

927

928

929

930

931

932

933

934

935

936

937

938

939

940

941

942

943

944

National Academy of Sciences, 105(33), 11969-11974.

https://doi.org/10.1073/pnas.0802569105

Stiers, P., Mennes, M., \& Sunaert, S. (2010). Distributed task coding throughout the multiple demand network of the human frontal-insular cortex. Neurolmage, 52(1), 252-262. https://doi.org/10.1016/j.neuroimage.2010.03.078

Stokes, M. G., Kusunoki, M., Sigala, N., Nili, H., Gaffan, D., \& Duncan, J. (2013). Dynamic Coding for Cognitive Control in Prefrontal Cortex. Neuron, 78(2), 364-375. https://doi.org/10.1016/j.neuron.2013.01.039

Vincent, J. L., Kahn, I., Snyder, A. Z., Raichle, M. E., \& Buckner, R. L. (2008). Evidence for a Frontoparietal Control System Revealed by Intrinsic Functional Connectivity. Journal of Neurophysiology, 100(6), 3328-3342. https://doi.org/10.1152/jn.90355.2008

Wagenmakers, E.-J. (2007). A practical solution to the pervasive problems ofp values. Psychonomic Bulletin \& Review, 14(5), 779-804. https://doi.org/10.3758/BF03194105

Waskom, M. L., Kumaran, D., Gordon, A. M., Rissman, J., \& Wagner, A. D. (2014). Frontoparietal Representations of Task Context Support the Flexible Control of GoalDirected Cognition. Journal of Neuroscience, 34(32), 10743-10755. https://doi.org/10.1523/JNEUROSCI.5282-13.2014

Wetzels, R., Matzke, D., Lee, M. D., Rouder, J. N., Iverson, G. J., \& Wagenmakers, E.-J. (2011). Statistical Evidence in Experimental Psychology: An Empirical Comparison Using 855 t Tests. Perspectives on Psychological Science, 6(3), 291-298. https://doi.org/10.1177/1745691611406923

White, A. L., \& Carrasco, M. (2011). Feature-based attention involuntarily and simultaneously improves visual performance across locations. Journal of Vision, 11(6), 15-15. https://doi.org/10.1167/11.6.15 
945 Woolgar, A., Afshar, S., Williams, M. A., \& Rich, A. N. (2015). Flexible Coding of Task Rules in Frontoparietal Cortex: An Adaptive System for Flexible Cognitive Control. Journal of Cognitive Neuroscience, 27(10), 1895-1911.

948 https://doi.org/10.1162/jocn_a_00827

Woolgar, A., Golland, P., \& Bode, S. (2014). Coping with confounds in multivoxel pattern analysis: What should we do about reaction time differences? A comment on Todd, Nystrom \& Cohen 2013. Neurolmage, 98, 506-512.

952 https://doi.org/10.1016/j.neuroimage.2014.04.059

Woolgar, A., Hampshire, A., Thompson, R., \& Duncan, J. (2011). Adaptive Coding of TaskRelevant Information in Human Frontoparietal Cortex. Journal of Neuroscience, 31(41), 14592-14599. https://doi.org/10.1523/JNEUROSCI.2616-11.2011

Woolgar, A., Jackson, J., \& Duncan, J. (2016). Coding of Visual, Auditory, Rule, and Response Information in the Brain: 10 Years of Multivoxel Pattern Analysis. Journal of Cognitive Neuroscience, 28(10), 1433-1454.

959 https://doi.org/10.1162/jocn_a_00981

Woolgar, A., Thompson, R., Bor, D., \& Duncan, J. (2011). Multi-voxel coding of stimuli, rules, and responses in human frontoparietal cortex. Neurolmage, 56(2), 744-752. https://doi.org/10.1016/j.neuroimage.2010.04.035 